\title{
Ecological Bistability and Evolutionary Reversals under Asymmetrical Competition
}

Fabio Dercole (dercole@elet.polimi.it)

Régis Ferrière (regis.ferriere@snv.jussieu.fr)

Sergio Rinaldi (rinaldi@elet.polimi.it)

\section{Approved by}

Ulf Dieckmann (dieckman@iiasa.ac.at)

Project Leader, Adaptive Dynamics Network

July 2002 


\section{IIASA STUDIES IN ADAPTIVE DYNAMICS No. 65}

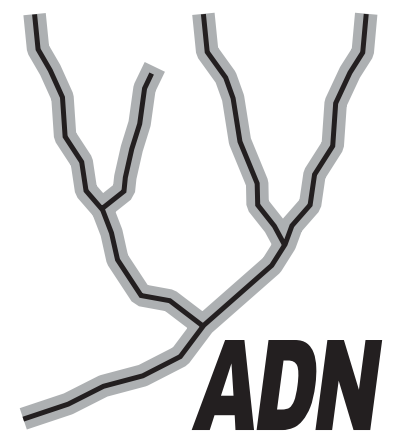

The Adaptive Dynamics Network at IIASA fosters the development of new mathematical and conceptual techniques for understanding the evolution of complex adaptive systems.

Focusing on these long-term implications of adaptive processes in systems of limited growth, the Adaptive Dynamics Network brings together scientists and institutions from around the world with IIASA acting as the central node.

Scientific progress within the network is collected in the IIASA Studies in Adaptive Dynamics series.

No. 1 Metz JAJ, Geritz SAH, Meszéna G, Jacobs FJA, van Heerwaarden JS: Adaptive Dynamics: A Geometrical Study of the Consequences of Nearly Faithful Reproduction. IIASA Working Paper WP-95-099 (1995). van Strien SJ, Verduyn Lunel SM (eds): Stochastic and Spatial Structures of Dynamical Systems, Proceedings of the Royal Dutch Academy of Science (KNAW Verhandelingen), North Holland, Amsterdam, pp. 183-231 (1996).

No. 2 Dieckmann U, Law R: The Dynamical Theory of Coevolution: A Derivation from Stochastic Ecological Processes. IIASA Working Paper WP-96-001 (1996). Journal of Mathematical Biology 34:579-612 (1996).

No. 3 Dieckmann U, Marrow P, Law R: Evolutionary Cycling of Predator-PreyInteractions: Population Dynamics and the Red Queen. IIASA Preprint (1995). Journal of Theoretical Biology 176:91-102 (1995).

No. 4 Marrow P, Dieckmann U, Law R: Evolutionary Dynamics of Predator-Prey Systems: An Ecological Perspective. IIASA Working Paper WP-96-002 (1996). Journal of Mathematical Biology 34:556-578 (1996).

No. 5 Law R, Marrow P, Dieckmann U: On Evolution under Asymmetric Competition. IIASA Working Paper WP-96-003 (1996). Evolutionary Ecology 11:485-501 (1997).

No. 6 Metz JAJ, Mylius SD, Diekmann O: When Does Evolution Optimize? On the Relation Between Types of Density Dependence and Evolutionarily Stable Life History Parameters. IIASA Working Paper WP-96-004 (1996).

No. 7 Ferrière R, Gatto M: Lyapunov Exponents and the Mathematics of Invasion in Oscillatory or Chaotic Populations. Theoretical Population Biology 48:126-171 (1995).

No. 8 Ferrière R, Fox GA: Chaos and Evolution. IIASA Preprint (1996). Trends in Ecology and Evolution 10:480485 (1995).

No. 9 Ferrière R, Michod RE: The Evolution of Cooperation in Spatially Heterogeneous Populations. IIASA Working Paper WP-96-029 (1996). The American Naturalist 147:692717 (1996).

No. 10 van Dooren TJM, Metz JAJ: Delayed Maturation in Temporally Structured Populations with Non-Equilibrium Dynamics. IIASA Working Paper WP-96-070 (1996). Journal of Evolutionary Biology 11:41-62 (1998).
No. 11 Geritz SAH, Metz JAJ, Kisdi É, Meszéna G: The Dynamics of Adaptation and Evolutionary Branching. IIASA Working Paper WP-96-077 (1996). Physical Review Letters 78:2024-2027 (1997).

No. 12 Geritz SAH, Kisdi É, Meszéna G, Metz JAJ: Evolutionary Singular Strategies and the Adaptive Growth and Branching of the Evolutionary Tree. IIASA Working Paper WP-96-114 (1996). Evolutionary Ecology 12:35-57 (1998).

No. 13 Heino M, Metz JAJ, Kaitala V: Evolution of Mixed Maturation Strategies in Semelparous Life-Histories: The Crucial Role of Dimensionality of Feedback Environment. IIASA Working Paper WP-96-126 (1996). Philosophical Transactions of the Royal Society of London Series B 352:1647-1655 (1997).

No. 14 Dieckmann U: Can Adaptive Dynamics Invade? IIASA Working Paper WP-96-152 (1996). Trends in Ecology and Evolution 12:128-131 (1997).

No. 15 Meszéna G, Czibula I, Geritz SAH: Adaptive Dynamics in a 2-Patch Environment: A Simple Model for Allopatric and Parapatric Speciation. IIASA Interim Report IR-97-001 (1997). Journal of Biological Systems 5:265-284 (1997).

No. 16 Heino M, Metz JAJ, Kaitala V: The Enigma of Frequency-Dependent Selection. IIASA Interim Report IR97-061 (1997). Trends in Ecology and Evolution 13:367-370 (1998).

No. 17 Heino M: Management of Evolving Fish Stocks. IIASA Interim Report IR-97-062 (1997). Canadian Journal of Fisheries and Aquatic Sciences 55:1971-1982 (1998).

No. 18 Heino M: Evolution of Mixed Reproductive Strategies in Simple Life-History Models. IIASA Interim Report IR-97063 (1997).

No. 19 Geritz SAH, van der Meijden E, Metz JAJ: Evolutionary Dynamics of Seed Size and Seedling Competitive Ability. IIASA Interim Report IR-97-071 (1997). Theoretical Population Biology 55:324-343 (1999).

No. 20 Galis F, Metz JAJ: Why Are There So Many Cichlid Species? On the Interplay of Speciation and Adaptive Radiation. IIASA Interim Report IR-97-072 (1997). Trends in Ecology and Evolution 13:1-2 (1998). 
No. 21 Boerlijst MC, Nowak MA, Sigmund K: Equal Pay for all Prisoners/ The Logic of Contrition. IIASA Interim Report IR-97-073 (1997). American Mathematical Society Monthly 104:303-307 (1997). Journal of Theoretical Biology 185:281-293 (1997).

No. 22 Law R, Dieckmann U: Symbiosis Without Mutualism and the Merger of Lineages in Evolution. IIASA Interim Report IR-97-074 (1997). Proceedings of the Royal Society of London Series B 265:1245-1253 (1998).

No. 23 Klinkhamer PGL, de Jong TJ, Metz JAJ: Sex and Size in Cosexual Plants. IIASA Interim Report IR-97-078 (1997). Trends in Ecology and Evolution 12:260-265 (1997).

No. 24 Fontana W, Schuster P: Shaping Space: The Possible and the Attainable in RNA Genotype-Phenotype Mapping. IIASA Interim Report IR-98-004 (1998). Journal of Theoretical Biology 194:491-515 (1998).

No. 25 Kisdi É, Geritz SAH: Adaptive Dynamics in Allele Space: Evolution of Genetic Polymorphism by Small Mutations in a Heterogeneous Environment. IIASA Interim Report IR-98-038 (1998). Evolution 53:993-1008 (1999).

No. 26 Fontana W, Schuster P: Continuity in Evolution: On the Nature of Transitions. IIASA Interim Report IR-98-039 (1998). Science 280:1451-1455 (1998).

No. 27 Nowak MA, Sigmund K: Evolution of Indirect Reciprocity by Image Scoring/ The Dynamics of Indirect Reciprocity. IIASA Interim Report IR-98-040 (1998). Nature 393:573-577 (1998). Journal of Theoretical Biology 194:561574 (1998).

No. 28 Kisdi É: Evolutionary Branching Under Asymmetric Competition. IIASA Interim Report IR-98-045 (1998). Journal of Theoretical Biology 197:149-162 (1999).

No. 29 Berger U: Best Response Adaptation for Role Games. IIASA Interim Report IR-98-086 (1998).

No. 30 van Dooren TJM: The Evolutionary Ecology of Dominance-Recessivity. IIASA Interim Report IR-98-096 (1998). Journal of Theoretical Biology 198:519-532 (1999).

No. 31 Dieckmann U, O'Hara B, Weisser W: The Evolutionary Ecology of Dispersal. IIASA Interim Report IR-98-108 (1998). Trends in Ecology and Evolution 14:88-90 (1999).

No. 32 Sigmund K: Complex Adaptive Systems and the Evolution of Reciprocation. IIASA Interim Report IR-98-100 (1998). Ecosystems 1:444-448 (1998).

No. 33 Posch M, Pichler A, Sigmund K: The Efficiency of Adapting Aspiration Levels. IIASA Interim Report IR-98103 (1998). Proceedings of the Royal Society London Series B 266:1427-1435 (1999).

No. 34 Mathias A, Kisdi É: Evolutionary Branching and Coexistence of Germination Strategies. IIASA Interim Report IR-99-014 (1999).

No. 35 Dieckmann U, Doebeli M: On the Origin of Species by Sympatric Speciation. IIASA Interim Report IR-99-013 (1999). Nature 400:354-357 (1999).

No. 36 Metz JAJ, Gyllenberg M: How Should We Define Fitness in Structured Metapopulation Models? Including an Application to the Calculation of Evolutionarily Stable Dispersal Strategies. IIASA Interim Report IR-99-019 (1999). Proceedings of the Royal Society of London Series B 268:499508 (2001)
No. 37 Gyllenberg M, Metz JAJ: On Fitness in Structured Metapopulations. IIASA Interim Report IR-99-037 (1999). Journal of Mathematical Biology 43:545-560 (2001).

No. 38 Meszéna G, Metz JAJ: Species Diversity and Population Regulation: The Importance of Environmental Feedback Dimensionality. IIASA Interim Report IR-99-045 (1999).

No. 39 Kisdi É, Geritz SAH: Evolutionary Branching and Sympatric Speciation in Diploid Populations. IIASA Interim Report IR-99-048 (1999).

No. 40 Ylikarjula J, Heino M, Dieckmann U: Ecology and Adaptation of Stunted Growth in Fish. IIASA Interim Report IR-99-050 (1999). Evolutionary Ecology 13:433-453 (1999).

No. 41 Nowak MA, Sigmund K: Games on Grids. IIASA Interim Report IR-99-038 (1999). Dieckmann U, Law R, Metz JAJ (eds): The Geometry of Ecological Interactions: Simplifying Spatial Complexity, Cambridge University Press, Cambridge, UK, pp. 135-150 (2000).

No. 42 Ferrière R, Michod RE: Wave Patterns in Spatial Games and the Evolution of Cooperation. IIASA Interim Report IR-99-041 (1999). Dieckmann U, Law R, Metz JAJ (eds): The Geometry of Ecological Interactions: Simplifying Spatial Complexity, Cambridge University Press, Cambridge, UK, pp. 318-332 (2000).

No. 43 Kisdi É, Jacobs FJA, Geritz SAH: Red Queen Evolution by Cycles of Evolutionary Branching and Extinction. IIASA Interim Report IR-00-030 (2000).

No. 44 Meszéna G, Kisdi É, Dieckmann U, Geritz SAH, Metz JAJ: Evolutionary Optimisation Models and Matrix Games in the Unified Perspective of Adaptive Dynamics. IIASA Interim Report IR-00-039 (2000).

No. 45 Parvinen K, Dieckmann U, Gyllenberg M, Metz JAJ: Evolution of Dispersal in Metapopulations with Local Density Dependence and Demographic Stochasticity. IIASA Interim Report IR-00-035 (2000).

No. 46 Doebeli M, Dieckmann U: Evolutionary Branching and Sympatric Speciation Caused by Different Types of Ecological Interactions. IIASA Interim Report IR-00-040 (2000). The American Naturalist 156:S77-S101 (2000).

No. 47 Heino M, Hanski I: Evolution of Migration Rate in a Spatially Realistic Metapopulation Model. IIASA Interim Report IR-00-044 (2000). The American Naturalist 157:495511 (2001).

No. 48 Gyllenberg M, Parvinen K, Dieckmann U: Evolutionary Suicide and Evolution of Dispersal in Structured Metapopulations. IIASA Interim Report IR-00-056 (2000). Journal of Mathematical Biology 45:79-105 (2002).

No. 49 van Dooren TJM: The Evolutionary Dynamics of Direct Phenotypic Overdominance: Emergence Possible, Loss Probable. IIASA Interim Report IR-00-048 (2000). Evolution 54: 1899-1914 (2000).

No. 50 Nowak MA, Page KM, Sigmund K: Fairness Versus Reason in the Ultimatum Game. IIASA Interim Report IR00-57 (2000). Science 289:1773-1775 (2000).

No. 51 de Feo O, Ferrière R: Bifurcation Analysis of Population Invasion: On-Off Intermittency and Basin Riddling. IIASA Interim Report IR-00-074 (2000). International Journal of Bifurcation and Chaos 10:443-452 (2000).

No. 52 Heino M, Laaka-Lindberg S: Clonal Dynamics and Evolution of Dormancy in the Leafy Hepatic Lophozia Silvicola. IIASA Interim Report IR-01-018 (2001). Oikos 94:525-532 (2001). 
No. 53 Sigmund K, Hauert C, Nowak MA: Reward and Punishment in Minigames. IIASA Interim Report IR-01-031 (2001). Proceedings of the National Academy of Sciences of the USA 98:10757-10762(2001).

No. 54 Hauert C, De Monte S, Sigmund K, Hofbauer J: Oscillations in Optional Public Good Games. IIASA Interim Report IR-01-036 (2001).

No. 55 Ferrière R, Le Galliard J: Invasion Fitness and Adaptive Dynamics in Spatial Population Models. IIASA Interim Report IR-01-043 (2001). Clobert J, Dhondt A, Danchin E, Nichols J (eds): Dispersal, Oxford University Press, pp. 57-79 (2001).

No. 56 de Mazancourt C, Loreau M, Dieckmann U: Can the Evolution of Plant Defense Lead to Plant-Herbivore Mutualism. IIASA Interim Report IR-01-053 (2001). The American Naturalist 158: 109-123 (2001).

No. 57 Claessen D, Dieckmann U: Ontogenetic Niche Shifts and Evolutionary Branching in Size-Structured Populations. IIASA Interim Report IR-01-056 (2001). Evolutionary Ecology Research 4:189-217 (2002).

No. 58 Brandt H: Correlation Analysis of Fitness Landscapes. IIASA Interim Report IR-01-058 (2001).

No. 59 Dieckmann U: Adaptive Dynamics of Pathogen-Host Interacations. IIASA Interim Report IR-02-007 (2002). Dieckmann U, Metz JAJ, Sabelis MW, Sigmund K (eds): Adaptive Dynamics of Infectious Diseases: In Pursuit of Virulence Management, Cambridge University Press, Cambridge, UK, pp. 39-59 (2002).
No. 60 Nowak MA, Sigmund K: Super- and Coinfection: The Two Extremes. IIASA Interim Report IR-02-008 (2002). Dieckmann U, Metz JAJ, Sabelis MW, Sigmund K (eds): Adaptive Dynamics of Infectious Diseases: In Pursuit of Virulence Management, Cambridge University Press, Cambridge, UK, pp. 124-137 (2002).

No. 61 Sabelis MW, Metz JAJ: Perspectives for Virulence Management: Relating Theory to Experiment. IIASA Interim Report IR-02-009 (2002). Dieckmann U, Metz JAJ, Sabelis MW, Sigmund K (eds): Adaptive Dynamics of Infectious Diseases: In Pursuit of Virulence Management, Cambridge University Press, Cambridge, UK, pp. 379-398 (2002).

No. 62 Cheptou P, Dieckmann U: The Evolution of SelfFertilization in Density-Regulated Populations . IIASA Interim Report IR-02-024 (2002). Proceedings of the Royal Society of London Series B 269:1177-1186 (2002).

No. 63 Bürger R: Additive Genetic Variation Under Intraspecific Competition and Stabilizing Selection: A Two-Locus Study. IIASA Interim Report IR-02-013 (2002). Journal of Theoretical Population Biology 61:197-213 (2002).

No. 64 Hauert C, De Monte S, Hofbauer J, Sigmund K: Volunteering as Red Queen Mechanism for Co-operation in Public Goods Games. IIASA Interim Report IR-02-041 (2002). Science 296:1129-1132 (2002).

No. 65 Dercole F, Ferrière R, Rinaldi S: Ecological Bistability and Evolutionary Reversals under Asymmetrical Competition. IIASA Interim Report IR-02-053 (2002). Evolution 56:1081-1090 (2002).

Issues of the IIASA Studies in Adaptive Dynamics series can be obtained at www.iiasa.ac.at/Research/ADN/Series.html or by writing to adn@iiasa.ac.at. 


\section{Contents}

1 Biological background $\quad 2$

2 Mathematical analysis $\quad 3$

2.1 The ecological model . . . . . . . . . . . . . . . . . . 3

2.2 Analysis of mutant-resident model . . . . . . . . . . . . . . . . . . 4

2.3 Adaptive dynamics model $\ldots \ldots \ldots \ldots \ldots$

3 Biological implications $\quad 7$

3.1 Predicting the occurrence of evolutionary reversals . . . . . . . . . . . . . 7

3.2 Effect of environmental change . . . . . . . . . . . . . . . . . . 9

4 Discussion 9

4.1 Mechanism causing evolutionary reversals . . . . . . . . . . . . . . 11

4.2 Concluding remarks . . . . . . . . . . . . . . . . . . . . . . 12

5 Appendix 18

5.1 Condition for ecological bistability . . . . . . . . . . . . . . . . . 18

5.2 Condition for adaptively-driven population cycles . . . . . . . . . . 18

5.3 Numerical analysis . . . . . . . . . . . . . . . . . . . . . . . . . 18 


\begin{abstract}
How does the process of life-history evolution interplay with population dynamics? Almost all models that have addressed this question assume that any combination of phenotypic traits uniquely determine the ecological population state. Here we show that if multiple ecological equilibria can exist, the evolution of a trait that relates to competitive performance can undergo adaptive reversals that drive cyclic alternation between population equilibria. The occurrence of evolutionary reversals requires neither environmentally-driven changes in selective forces, nor the co-evolution of interactions with other species. The mechanism inducing evolutionary reversals is two-fold. First, there exist phenotypes near which mutants can invade and yet fail to become fixed; although these mutants are eventually eliminated, their transitory growth causes the resident population to switch to an alternative ecological equilibrium. Second, asymmetrical competition causes the direction of selection to revert between high and low density. When ecological conditions for evolutionary reversals are not satisfied, the population evolves toward a steady state of either low or high abundance, depending on the degree of competitive asymmetry and environmental parameters. A sharp evolutionary transition between evolutionary stasis and evolutionary reversals and cycling can occur in response to a smooth change in ecological parameters, and this may have implications for our understanding of size-abundance patterns.
\end{abstract}




\author{
About the Authors \\ Fabio Dercole \\ CIRITA, Politecnico di Milano \\ Via Ponzio 34/5, 20133 Milano, Italy \\ Régis Ferrière \\ Mathematical Eco-Evolutionary Theory (MEET) Group \\ Laboratoire d'Ecologie, Ecole Normale Supérieure \\ 46 rue d'Ulm, 75230 Paris cedex 05, France \\ and \\ Adaptive Dynamics Network \\ International Institute for Applied Systems Analysis \\ A-2361 Laxenburg, Austria \\ Sergio Rinaldi \\ CIRITA, Politecnico di Milano \\ Via Ponzio 34/5, 20133 Milano, Italy
}

\title{
Acknowledgement
}

The authors are very grateful to Peter Abrams for his comments and constructive criticisms. F. D. is indebted to the International Institute for Applied Systems Analysis (Laxenburg, Austria) for allowing him to participate in the Young Scientist Summer Program 2000. Financial support was received from CESTIA-CNR, Politecnico di Milano, Italy, and from the European Research Training Network ModLife, funded through the Human Potential Programme of the European Commission (Contract HPRN-CT-2000-00051). 


\title{
Ecological Bistability and Evolutionary Reversals under Asymmetrical Competition
}

\author{
Fabio Dercole \\ Régis Ferrière \\ Sergio Rinaldi
}

The contrasting patterns of evolution in populations with chronically high or low densities have captured the shared interests of ecologists and geneticists for a long time (Travis, 1990). There has been a considerable amount of verbal and mathematical theory on the subject (reviewed lucidly by Mueller (1997)). In this context, the integrative notion of an eco-evolutionary feedback emphasizes that selective pressures exerted on individuals are shaped by population structure and abundance; in return, population dynamics are primarily determined by individual phenotypic traits that are genetically-based and molded by natural selection (Pimentel, 1968; Heino et al., 1998).

Density-dependent selection is the central theme underlying one arch of the eco-evolutionary feedback - from ecology to evolution. Over the last two decades, there has been intensive empirical investigation of the effect of population density on the nature, direction and strength of selection on life-history traits. Exemplary studies include those by Wilbur (1984) and Travis et al. (1985) on larval anurans, who showed that the role of predatory salamanders as a selective force for rapid growth of tadpoles varies depending on tadpole density: at very low densities this force is weak because growth rates are high and encounter rates with predators are low; greater numerical impact of predators occur only at higher tadpole densities; yet at extremely high densities all tadpoles grow so slowly, due to effective food limitation, that they can be driven extinct. The net result is that effective selection on growth rate will occur only within certain regions of the parameter space, depending upon the corresponding population density.

Although much empirical effort has been devoted to understand how crowding influences patterns of selection on life-history traits, the backward effect of selection on population dynamicsthe other arch of the eco-evolutionary feedback-remains poorly understood, even theoretically (Metz et al., 1992; Rand et al., 1994; Heino et al., 1998). One important feature of densitydependent models of population dynamics is their propensity to generate complex dynamics, including periodic and chaotic oscillations. Much attention has been paid to the question of whether natural selection could drive individual traits from values leading to stable equilibria, to values corresponding to cycles or chaos (Gatto, 1993; Ferrière and Gatto, 1993, 1995; Doebeli and Koella, 1995; Ferrière and Fox, 1995; Ebenman et al., 1996); contrasting theoretical predictions are awaiting to be tested empirically. A crucial assumption in all models developed around this issue is that any combination of traits uniquely determines the population state (De Feo and Ferrière, 2000). However, ecological models can have alternative equilibria (see Scheffer et al., 2001, for a survey), associated with different equilibrium densities, that can be reached for the very same individual trait values. The question of how the eco-evolutionary feedback works in these cases is basically untouched and provides the motivation for this work. 
We consider a very simple population model that can have a high and a low density equilibrium. At the former, there is selection for a trait that reduces population density, but at the latter there is selection for a trait that increases population density. Under some conditions that we identify by means of the adaptive dynamics approach (Metz et al., 1992; Geritz et al., 1997), these opposing patterns of selection result in periodic evolutionary reversals. Our analysis also predicts the influence of a set of environmental parameters on the outcome, and investigates the full interplay of population density and selective pressures that result from the eco-evolutionary feedback. A salient result of our study is that there are broad ecological conditions under which the direction of natural selection periodically reverts in conjunction with switches between ecological equilibria.

\section{Biological background}

We consider a single species whose population dynamics are determined by a set of fixed ecological parameters, and by life-history parameters that are functions of a phenotypic trait. This trait is assumed to be genetically determined and heritable, but it may vary among individuals due to mutations of small effects. Selection acts upon this variation, with changes in population dynamics as a potential by-product. We assume that for suitable environmental parameters there can be values of the trait for which the population possesses alternative stable equilibria.

Ecological bistability was first put forward by Holling (1973), Noy-Meir (1975), and May (1977). Subsequently, alternative low- and high- density states have been found in a host of ecological models. Although the first experimental examples that were proposed were criticized strongly (Connell and Sousa, 1983), more recent studies (reviewed in Scheffer et al. (2001)) support the view that multiple stable states can characterize natural systems.

Here we assume a simple phenomenological model to generate alternative stable equilibria, akin to the minimal models proposed by Scheffer et al. (2001). As explained below, one possible interpretation of this model is that the species under consideration is harvested by a generalist predator, which exploits a spectrum of other prey species. This assumption ensures that potential variations in selective pressures derive exclusively from intraspecific interactions.

We also assume that the phenotypic trait is related to competitive performance, and we call it, for this reason, 'competitive ability'. Thus, variation in individual trait results in asymmetrical competition between phenotypes. Moreover, individuals that are at competitive advantage are none-the-less expected to suffer some physiological cost: this is why competitive ability is assumed to be costly to some components of reproductive success. A common case involving asymmetrical competition and physiological costs of higher energy demands occurs when competing individuals differ in size. Often, larger individuals enjoy a competitive advantage because of their superior ability at obtaining limited resources (Brooks and Dodson, 1965; Wilson, 1975; Persson, 1985; Calder III, 1996), but the opposite can also be true, like in the case of small zooplanktivorous fish outcompeting large ones for food (Persson et al., 1998). However, this dichotomy does not influence our findings, since the only assumption that counts in this theory is that a positive variation of the trait is accompanied by an advantage in competition (or growth) and by a counterbalancing disadvantage in growth (or competition).

The body size interpretation of competitive ability may be useful because the relationship between body size and population abundance arguably has attracted great attention. Yet the search for general patterns has not been conclusive (Lawton, 1989; Blackburn and Lawton, 1994; Rosenzweig, 1995). Data from extant populations show that low abundance, under some circumstances, is associated with large body size (Brown, 1995; Gaston and Kunin, 1997), but the pattern is far from general. For example, Navarette and Menge (1997) found no tendency for small species to occur at higher densities than larger species in the tropical intertidal communities of Panama, 
in sharp contrast with the pattern in intertidal communities in temperate Chile (Marquet et al., 1990). Thus, although direct measures of competitive performance are difficult to obtain from real systems, there is hope that our theoretical predictions could be tested against the results of experimental studies (like those reported by Lenski and Bennett (1993) for bacteria) on the evolution of body size.

\section{Mathematical analysis}

\subsection{The ecological model}

We describe the ecological dynamics of the population of a single competition phenotype $u$ by making use of the following simple model:

$$
\dot{x}=G(x, u) x-H(x)
$$

where $x$ denotes population density. The function $G$ measures the logistic component of population growth; it is given by: $G(x, u)=r(u)-a x$, where $r(u)$ is the per capita intrinsic growth rate which is assumed to decrease with competitive ability $u$ to express the cost of enhanced competitive performance and $a x$ measures the extra mortality caused by intraspecific competition. Competitive ability $u$ scales between 0 and 1 . This scaling is achieved by means of the transformation $u=\log \left(l / l_{\min }\right) / \log \left(l_{\max } / l_{\min }\right)$, where $l$ is the 'real' trait value, and $l_{\min }$ and $l_{\max }$ are minimum and maximum trait values, respectively (Schwinning and Fox, 1995).

Bistability is triggered by combining the negative density-dependence of logistic growth with the positive density-dependence of an additional demographic factor. Two important examples of such a factor are: the reduction of reproduction due to a shortage of mating encounters in sparse population; and the reduction of mortality due to the dilution of predation risk as density increases (Dennis (1989) and references therein; Stephens and Sutherland (1999)). Both examples can be accounted for by the same model, namely a discount term $H(x)=x c /(x+d)$ in equation (1) (hereafter $H$ will be refered to as 'discounting function'). This form of $H$ reflects a negative exponential distribution of mating encounters (Dennis, 1989), or a Holling type-II functional response of the predator (May, 1977; Huberman, 1978; Brauer, 1979). The resulting population model leads, under suitable conditions on parameters, to the coexistence of two stable equilibria: the trivial equilibrium of extinction, and a positive equilibrium.

In this paper we investigate a more general scenario of ecological bistability involving the coexistence of two non-trivial stable equilibria. This is obtained simply by altering the discounting function $H$ into $H(x)=x c x /\left(x^{2}+d^{2}\right)$ which provides a simple way of accounting for spatial heterogeneity in predation risk or the chance of mating. With this choice of $H$, model (1) predicts the existence of a lower threshold on competitive ability, $u_{l}$, below which the population assumes a single stable equilibrium at high density; and an upper threshold, $u_{h}$, above which the population possesses a single stable equilibrium at low density (Appendix). For $u$ values lying between $u_{l}$ and $u_{h}$, there are two alternative stable equilibria, one corresponding to a state of high abundance (denoted by $\bar{x}$ ) and the other to a state of low abundance $(\underline{x})$, see Figure 1 .

It is also worth noticing that if body size is a measure of competitive ability, and the discounting function $H$ is predation rate, it would be more realistic to assume that $H$ in (1) depends also upon $u$ since prey body size usually influences the functional response of the predator. However, this would give rise to an analytically intractable problem even if the final result would be qualitatively the same. For this reason we have assumed that $H$ is independent on $u$. In any case, model (1) applies when competitive ability does not influence predation or when body size has only a weak influence on the predator functional response. 


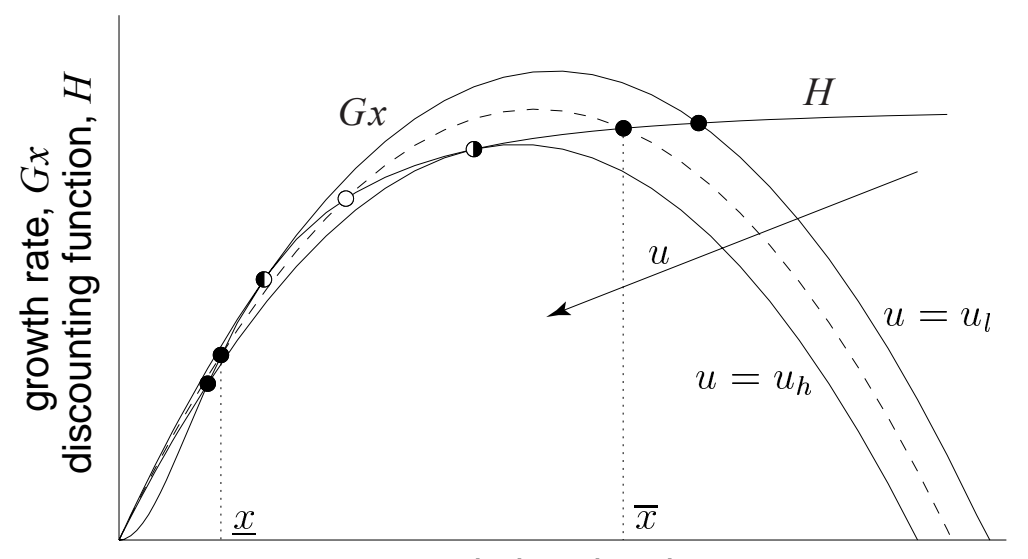

population density, $x$

Figure 1: Multiple ecological equilibria. Population equilibria are given by the intersection of the population growth function $G x$ (which depends on phenotypic trait $u$ ) and the discounting function $H$. For $u$ between the lower threshold $u_{l}$ and the upper threshold $u_{h}$ (see dashed line), the population has three ecological equilibria: two stable equilibria (filled circles), one at low population density $\underline{x}$ and the other at high population density $\bar{x}$; and one unstable equilibrium (open circle). For the two critical values of $u$, namely $u_{l}$ and $u_{h}$, there is a collision of a stable equilibrium with an unstable equilibrium (see half-filled circles).

\subsection{Analysis of mutant-resident model}

Having this ecological model in place, we describe the evolutionary dynamics of the individual trait $u$ that we regard as adaptive. Adaptive evolution is assumed to proceed by discrete steps involving small random mutations, and selection (Metz et al., 1992; Geritz et al., 1997). Mutations are assumed to be sufficiently rare so that two conditions are fulfilled: $(i)$ at the time a mutant phenotype arises, the resident population of the wild type is at ecological equilibrium, and ( $i i)$ the wild type population is challenged by one mutant phenotype at a time. Once the mutant has entered the population, it may simply go extinct, or invade and go to fixation by replacing (or coexisting with) the resident type, thereby setting the new wild environment against which the next mutant will have to strive. These two conditions amount to separating out the ecological and evolutionary timescales; they allow us to describe the evolutionary dynamics of the adaptive trait $u$ as a random walk, each step of which being taken according to the outcome of competition between a resident population and a mutant population (Champagnat et al., 2001).

The dynamics of interacting resident and mutant populations characterized by traits $u$ and $u^{\prime}$, respectively, are governed by the following system

$$
\begin{aligned}
& \dot{x}=g\left(x, x^{\prime}, u, u^{\prime}\right) x-h\left(x, x^{\prime}\right) \\
& \dot{x^{\prime}}=g\left(x^{\prime}, x, u^{\prime}, u\right) x^{\prime}-h\left(x^{\prime}, x\right)
\end{aligned}
$$

We assume that there is no discrimination between resident and mutant populations with regard to discounting (e. g. no discrimination by a predator), so that the function $h$ is given by

$$
h\left(x, x^{\prime}\right)=x \frac{c\left(x+x^{\prime}\right)}{\left(x+x^{\prime}\right)^{2}+d^{2}}
$$

By contrast, the per capita growth function $g$ must incorporate the effect of asymmetrical competition between two phenotypes. This is achieved by setting

$$
g\left(x, x^{\prime}, u, u^{\prime}\right)=r(u)-a x-b\left(u, u^{\prime}\right) x^{\prime}
$$


The competition coefficient $b$ is taken to depend only on the difference between phenotypic values $u^{\prime}$ and $u$ and is equal to $a$ for $u^{\prime}=u$. It should increase as the difference $u^{\prime}-u$ increases, expressing a stronger deleterious effect on the $u$-population growth of larger $u^{\prime}$-competitors. The slope of this function as $u^{\prime}-u$ goes to zero provides a measure of the strength of the asymmetry, and is denoted by $\beta$ in the following, i. e.

$$
\beta=\left.\frac{\mathrm{d} b}{\mathrm{~d}\left(u^{\prime}-u\right)}\right|_{u^{\prime}=u}
$$

All possible outcomes of resident-mutant competition can be classified by means of a detailed analysis of system (2-4) in the trait space $\left(u, u^{\prime}\right)$. In principle, this analysis could be carried out through extensive simulation of system (2-4) for various values of $u$ and $u^{\prime}$. However, in practice, the simulation approach is rather critical. This is why a much more effective method, called bifurcation analysis (Kuznetsov, 1998), has been used in this paper. The assumption that mutations have small effects makes it legitimate to restrict the analysis to the vicinity of the diagonal line $u=u^{\prime}$, that is, to the region of the trait space where the mutant phenotype differs only slightly from the resident. The result is shown in Figure 2, which unravels seven regions corresponding to seven qualitatively different behaviors of the dynamical system (2-4). In region I, the resident population possesses only one stable equilibrium, namely the high-density equilibrium $\bar{x}$. Small mutations characterized by slightly larger phenotype invade and replace the resident type (side panel I). The evolutionary random walk takes on a small step toward larger phenotype, and this results in a slight decrease of the equilibrium population density (see Fig. 1). The same outcome is observed in all regions II-V (side panels II-V). In all of these regions, a mutant with a slightly larger phenotype can invade and eliminate the resident type. Thus, starting from any ancestral state of high abundance for which $u$ is less than $u_{h}$, adaptive evolution proceeds through the gradual increase of the phenotype, causing the concurrent, slow decrease of population density.

A new phenomenon is observed in region VI, where the resident trait is still slightly less than $u_{h}$ while the mutant trait is now slightly greater than $u_{h}$. In this case, the mutant first invades, but the resident 'strikes back' (side panel VI): after its initial increase, the mutant reaches a peak density, then it starts declining and eventually goes extinct. This unexpected dynamical pattern arises from the existence, for the resident population, of two alternative equilibria which differ in their vulnerability to invasion by the mutant. Initially the resident population stands on its high-density equilibrium, which appears to be invasible. As the mutant population grows, the resident population cannot sustain its high density any longer and starts to approach its low-density state. While doing so, it creates a competitive environment that becomes non-invadable by the mutant. Now the latter is doomed, and as it goes extinct the population is sent back to its original phenotypic state; but in the mean time the ecological state has changed: the resident population experienced a sudden transition from the state of high abundance to the state of low abundance.

The same picture holds for the dual case, starting from an ancestral state of low abundance and intermediate or large phenotype ( $u$ larger than $u_{l}$ ). Here the adaptive process drives the slow decrease of the trait and the concomitant slight increase of population density. When the phenotypic state comes close to the low threshold $u_{l}$, any invasion attempt by mutants smaller than $u_{l}$ triggers the ecological switch of the resident population from $\underline{x}$ to $\bar{x}$. Altogether this analysis shows that the evolutionary dynamics of the population remains monomorphic and never comes to a halt. The phenotypic state of the population oscillates between a minimum $\left(u_{l}\right)$ and a maximum $\left(u_{h}\right)$ trait value. The ecological state of the population switches between low and high densities every time the adaptive process reaches either of these threshold phenotypes. 


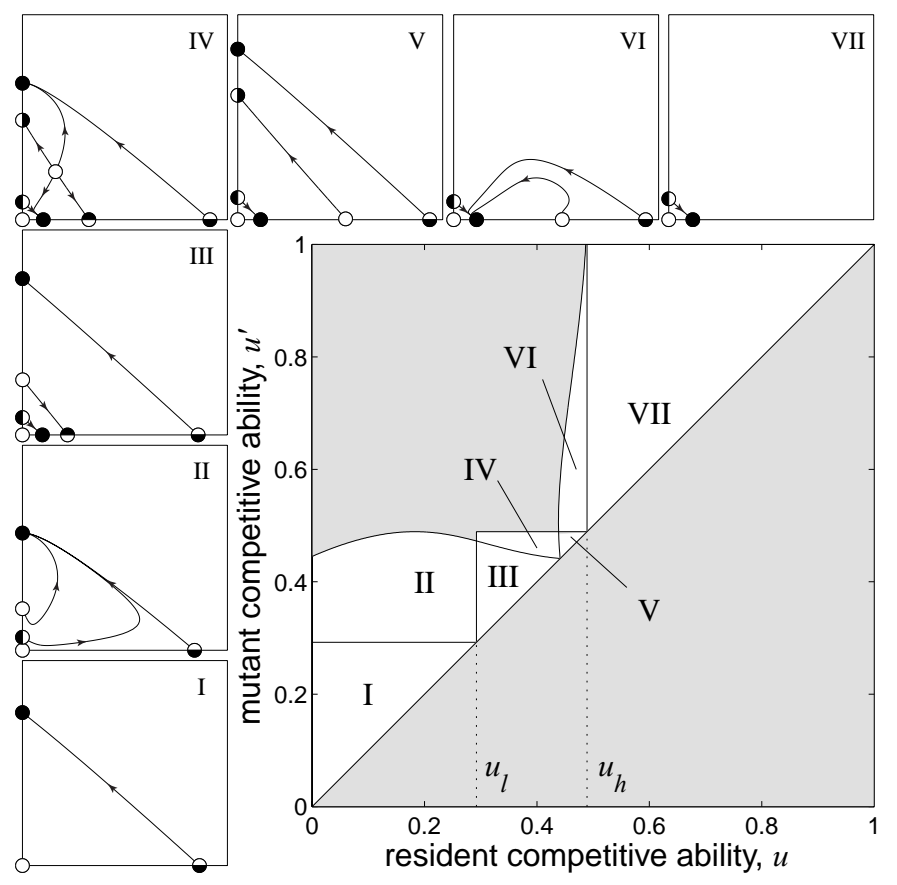

Figure 2: Bifurcation diagram of the resident-mutant model (2-4) in the resident-mutant trait space $\left(u, u^{\prime}\right)$. The bifurcation diagram (main panel) is symmetrical with respect to the diagonal line; only its upper part is shown. Mutant trait values must be close to resident trait values (under the assumption of small mutational effects), therefore the bifurcation analysis is restricted to the neighborhood of the diagonal. Seven regions (I-VII) correspond to qualitatively different behaviors for the resident-mutant system. A side panel that describes the population dynamics in the state space $\left(x, x^{\prime}\right)$ is attached to each region. In these panels, filled circles indicate stable equilibria, open circles: unstable equilibria, half-filled circles: saddle points. Resident-mutant trait values in side panels: $(0.1,0.2)$ in panel I, $(0.2,0.4)$ in II, $(0.35,0.4)$ in III, $(0.4,0.48)$ in IV, $(0.46,0.48)$ in V, $(0.46,0.6)$ in VI, $(0.6,0.7)$ in VII. Other parameters: $a=0.1, \beta=0.33, c=1.0$, $d=0.75, r_{0}=1.0, \gamma=1.0$ (see Appendix for a full specification of the model). 


\subsection{Adaptive dynamics model}

One can achieve similar conclusions by using an adaptive dynamics model which provides a deterministic approximation of the mutation-selection random walk process described above (Dieckmann and Law, 1996). The adaptive dynamics model reads:

$$
\begin{aligned}
& \dot{x}=G(x, u) x-H(x) \\
& \dot{u}=k x s(u, x)
\end{aligned}
$$

This model gives the rate of change $\dot{u}$ of the adaptive trait $u$ as a function of population density $x$ and of the selection gradient denoted by $s(u, x)$, scaled by a constant rate of evolutionary innovation, $k$. The selection gradient is defined as the slope of the fitness landscape in the vicinity of the resident phenotype, and is therefore given by

$$
s(u, x)=\left.\frac{\partial}{\partial u^{\prime}} g\left(x^{\prime}, x, u^{\prime}, u\right)\right|_{u^{\prime}=u, x^{\prime}=0}
$$

The dynamics governed by system (5) involve a fast component, that of population dynamics governed by the first equation; and a slow component, that of adaptive dynamics described by the second equation. The analysis of such a slow-fast system can be performed through a method popularized in ecology by May (1977) (see also Rinaldi and Scheffer (2000) for a recent survey and Matsuda and Abrams (1994b) and Khibnik and Kondrashov (1997) for applications in the context of eco-evolutionary dynamics). The analysis requires two steps. First, the two stable equilibria $\bar{x}(u)$ and $\underline{x}(u)$ of the fast component are determined for each frozen value of the slow variable $u$. This yields the thin curves shown in the first row of Figure 3. Then the sign of $\dot{u}$ at each point $(u, \bar{x}(u))$ and $(u, \underline{x}(u))$ is determined so as to predict the direction of phenotypic change. This amounts merely to finding the isocline $s(u, x)=0$ in the space $(u, x)$. Figure 3 displays the three possible outcomes. In Figure 3A, starting from point 0, we observe a first, fast population transient $(0 \rightarrow 1)$, followed by a slow evolutionary transient $(1 \rightarrow 2)$, entraining a slow ecological change yet leaving the population with low abundance. At point 2, a fast transient of ecological change abruptly brings the population to high abundance $(2 \rightarrow 3)$, and from point 3 a final slow evolutionary transient takes place, driving the population toward an eco-evolutionary equilibrium at point 4 where the phenotype is associated with high population density. At this point, selection may turn disruptive, causing evolutionary branching and the further evolution of polymorphism (Kisdi, 1999), but the investigation of this phenomenon lies outside the scope of the present article. Figure 3B shows a similar pattern whereby the evolutionary process drives the population toward a state of permanent low abundance and promotes large phenotypes.

In contrast, the eco-evolutionary process never comes to a halt in the case shown in Figure 3C. Here, after a first, fast ecological transient $(0 \rightarrow 1)$, and a slow evolutionary transient $(1 \rightarrow 5)$, the system is trapped forever on a slow-fast limit cycle $(5 \rightarrow 2 \rightarrow 3 \rightarrow 4 \rightarrow 5)$. One can see by comparing Figures $3 \mathrm{~A}, \mathrm{~B}$, and $\mathrm{C}$ that the necessary and sufficient condition for the existence of such periodic evolutionary reversals is that the selection isocline $s(u, x)=0$ (boundary of grey regions in Fig. 3) separates the two stable branches of equilibria of the population. This is a general condition for the existence of slow-fast limit cycles, known as separation principle (Muratori and Rinaldi, 1991).

\section{Biological implications}

\subsection{Predicting the occurrence of evolutionary reversals}

The occurrence of evolutionary reversals and adaptively-driven population cycles depends on four ecological parameters: the intraspecific competition coefficient, $a$, the strength of intraspecific 

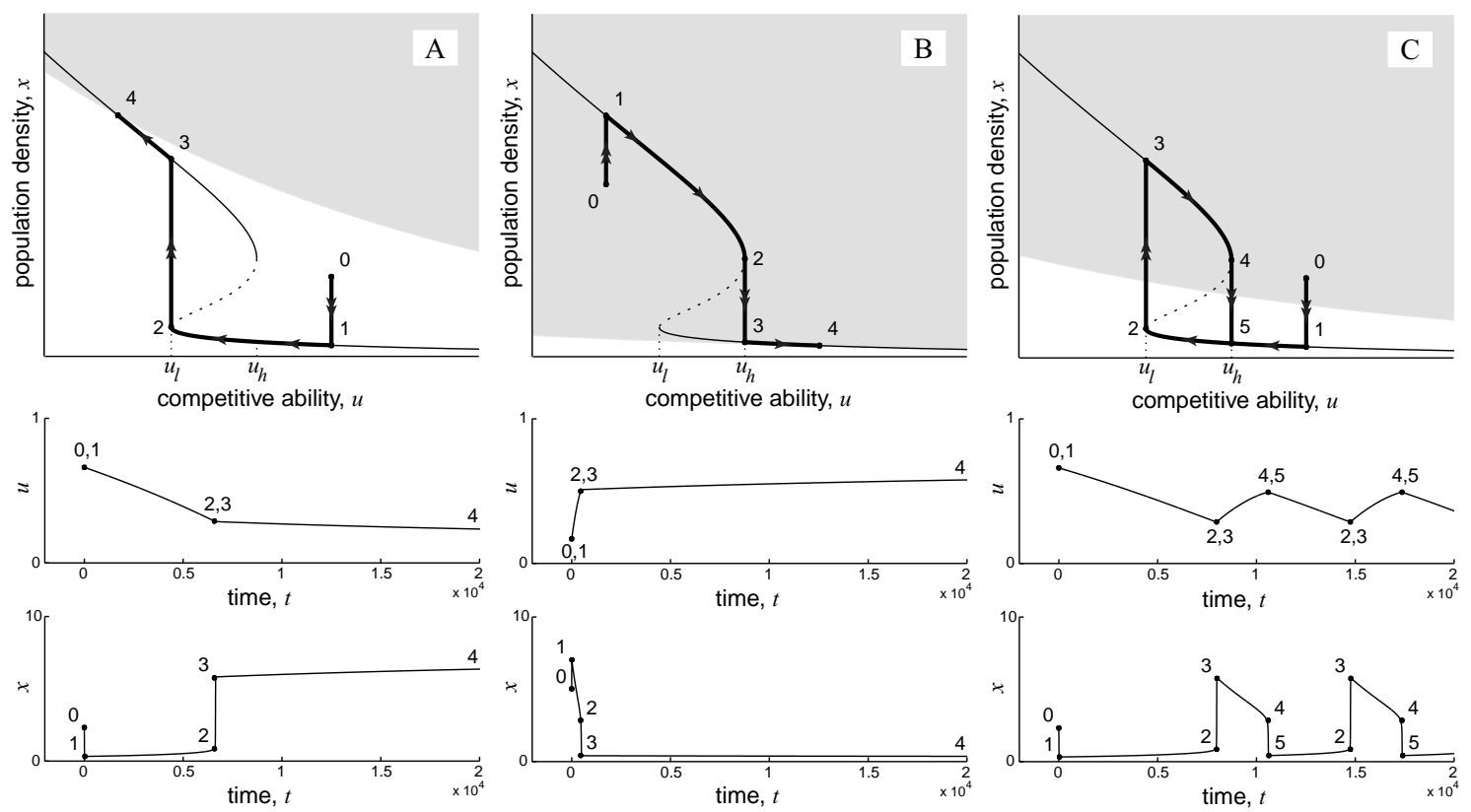

Figure 3: Eco-evolutionary dynamics in the space of trait $u$ and population density $x$ (first row) and corresponding time evolution of $u$ and $x$ (second and third rows). In the first row, thin curves indicate the set of equilibrium population density as a function of the trait value $u$ (plain portions: stable equilibria, dotted portions: unstable equilibria); shaded region: positive selection gradient $(s(u, x)>0)$; thick curve: exemplary trajectory (single arrows: slow dynamics, double arrows: fast dynamics). A, Evolution toward small trait and permanent high abundance $(\beta=0.12)$. B, Evolution toward large trait and permanent low abundance $(\beta=1.6)$. $\mathbf{C}$, Periodic evolutionary reversals and adaptively-driven population cycle $(\beta=$ $0.33)$. Other parameter values as in Figure 2, and $k=10^{-4}$. 
competition asymmetry, $\beta$, and the discount parameters, $c$ and $d$. Notice that in the case where discounting is due to predation, $c$ and $d$ measure the predation rate and the predator half-saturation constant, respectively. Evolutionary reversals can develop only when the population possesses two alternative ecological equilibria, which imposes the condition $a<c / 8 d^{2}$ (Appendix and Fig. 4A). Thus, ecological bistability requires that the competition coefficient be lower than a threshold set by the parameters of the discounting function; this constraint becomes weaker as the discounting pressure increases (larger $c$ and/or smaller $d$ ).

In ecologically bistable populations, the occurrence of periodic evolutionary reversals is determined by the strength of competitive asymmetry, which has to lie within a range of intermediate values such that selective pressures on the adaptive trait revert at low and high abundance (Appendix). Figures $4 \mathrm{~A}$ and $\mathrm{B}$ show that this range shrinks under harsher conditions expressed by a larger competition coefficient $a$, whereas for fixed $a$, it expands if discounting is enhanced (larger $c$ and/or smaller $d$ ). Altogether, a consistent pattern is that evolutionary reversals are more likely to occur in species characterized by significant competitive asymmetry, and facing strong discounting pressure.

When conditions for periodic evolutionary reversals are not satisfied, permanent low abundance and permanent high abundance represent alternative by-products of natural selection operating on competitive ability. Although there is a narrow range of parameters for which permanent high abundance evolves concomitantly with intermediate phenotypic value (Fig. 4C), there is a clear tendency for high abundance to evolve most often along with small trait value, whereas permanent low abundance can be associated with the evolution of large as well as intermediate trait value. This may help understand why the association of rarity and large body size in groups of closely related species remains difficult to establish empirically.

\subsection{Effect of environmental change}

The ecological parameters $a, \beta, c, d$ may vary in response to environmental change. It turns out that even small and smooth changes in these parameters may have a dramatic impact on the ecoevolutionary dynamics of the population. Here we focus our discussion on the transition between eco-evolutionary cycles and permanent low abundance. A transition of this kind caused by a slight environmental deterioration should imply a dramatic rise of the extinction risk. Population cycling may suddenly lose evolutionary stability and be replaced with a state of permanent low abundance when selection at low density ceases to be directional (toward the critical, low trait value at which adaptive reversal occurs) and becomes stabilizing instead (transition seen from Fig. 3C to B). This can happen as a result of (i) a small increase in competitive asymmetry, $\beta$, which makes the direction of selection on any trait value change at some lower population density actually reached in the state of low abundance; (ii) slightly more competition (larger competition coefficient $a$ ), or slightly less discounting (small decrease of $c$, or small increase of $d$ ), which cause the whole range of equilibrium population density across the state of low abundance to rise, leading the selective pressure induced by competitive asymmetry to dominate and favor larger phenotype, hence permanent low density.

\section{Discussion}

How density can influence and be influenced by evolutionary processes has concerned biologists ever since Darwin suggested that abundant species were more likely than rare species to be the sources of evolutionary novelties. Nonetheless, despite considerable attention to the matter, evolutionary biologists still hold widely varying views on the consequences of population density on evolution (Orians, 1997), and on the role of evolution in determining population abundance and 

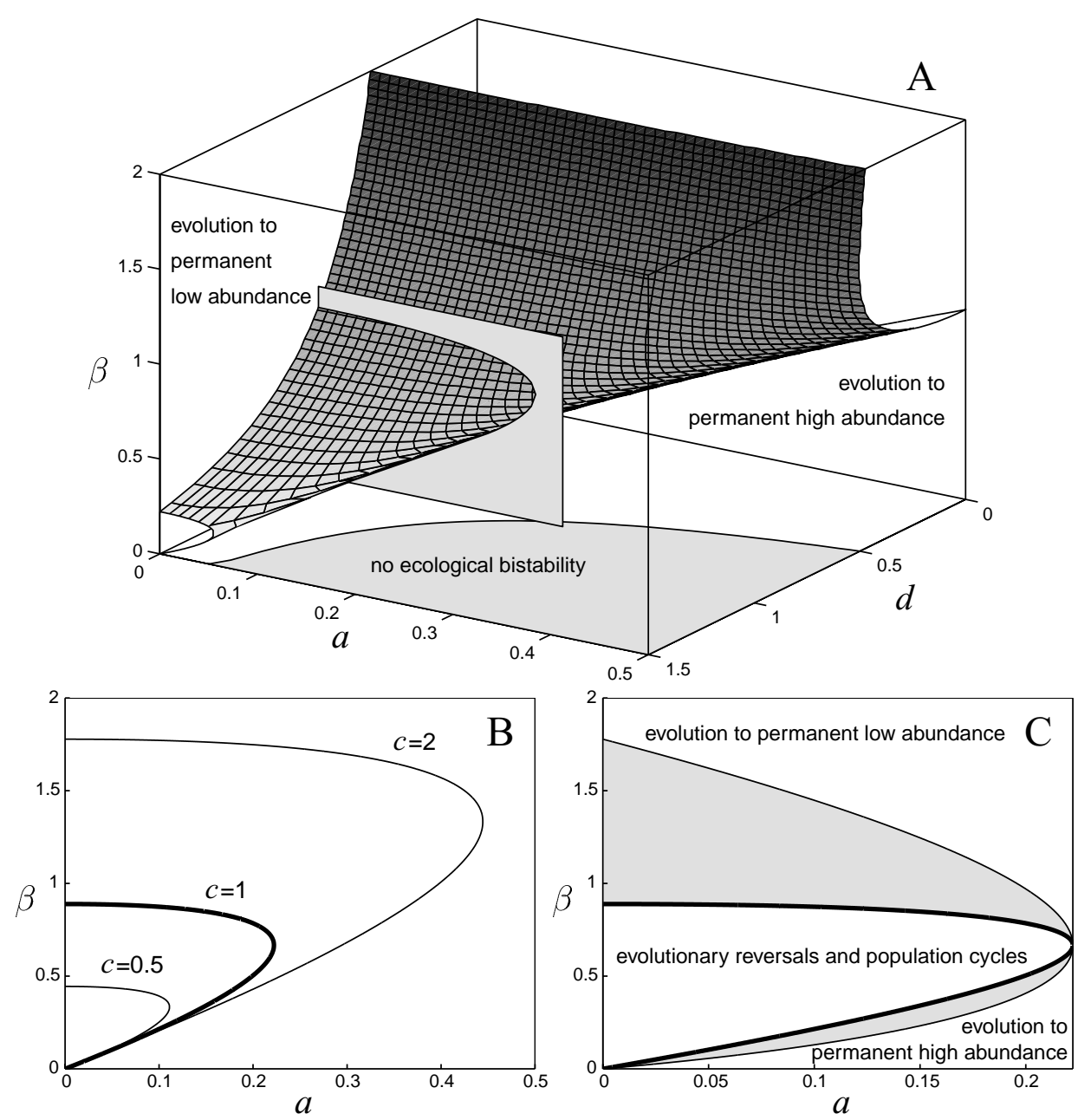

Figure 4: Eco-evolutionary dynamics depending on ecological parameters $a$ (coefficient of intraspecific competition), $\beta$ (strength of competitive asymmetry), and $c$ and $d$ (discounting parameters). A, Effect of $a, d$ and $\beta$. The volume bounded by the displayed surface contains parameter combinations that lead to periodic evolutionary reversals. Permanent low abundance evolves above the surface. Permanent high abundance evolves below the surface. Ecological bistability occurs only for values of $a$ and $d$ lying outside the shaded region in the horizontal plane. Other parameter values: $c=1.0, r_{0}=1.0, \gamma=1.0$. B, Effect of discounting coefficient $c$ on the region (inside curves) of $a$ and $\beta$ values for which periodic evolutionary reversals occur $(d=0.75)$. C, Effect of $a$ and $\beta$ on the transition between adaptively-driven population cycles and either permanent low abundance with large phenotypic trait, or permanent high abundance and small phenotypic trait. The shaded region indicates parameter combinations for which permanent low abundance and permanent high abundance evolve along with intermediate trait value. In $\mathbf{B}$ and $\mathbf{C}$, the bold curve bounds the $(a, \beta)$ region where periodic evolutionary reversals develop, and corresponds to the cross section indicated in $\mathbf{A}$. 
dynamics (Holt, 1997). This theoretical study investigates the interaction between population density and life-history evolution by focusing on an individual trait related to competitive performance that is potentially under different selection pressures depending on the population abundance. Our model allows for multiple ecological equilibria, which entails that the population may rest on a state of low abundance or on a state of high abundance under the very same conditions.

potentially under different selection pressures depending on In ecological systems that possess alternative equilibria, the equilibrium on which the population settles depends on ecological history, and recurrent jumps between equilibria have been traditionally explained by means of environmental or ecological factors (e. g., Southwood and Comins, 1976; Hanski, 1985; Crawley, 1992). In contrast, our analysis shows that the periodic alternation between low-density and highdensity equilibria can develop on the evolutionary timescale as a result of a purely endogenous mechanism: periodic reversals of selection on a phenotypic trait.

The simple, deterministic model considered here produces perfectly regular evolutionary cycles in trait value and population density. Random variability, however, is inherent to all populations. In a stochastic environment, the regularity of such slow-fast cycles is likely to break down (Rinaldi and Scheffer, 2000), in lieu of which more irregular alternations of phases of low and high abundance should be expected. Documenting such fluctuations in population abundance requires exceptional data sets collected on a timescale seldom accessible to ecologists. Perhaps the best example of such data is provided by subfossil deposits of pelagic fish, which have been used to reconstruct several millenia of population dynamics, with time resolution as fine as a decade (Soutar and Isaacs, 1974; Baumgartner et al., 1992). In the Pacific sardine Sardinops caerulea, population data show that the species has persisted at very low density over more than $55 \%$ of the time, through phases of irregular durations, from 10-200 years. Alternations between low and high abundance occurred rapidly and more or less in unison across the whole species range. Interestingly, a competitor, and possibly predator species, the Northern anchovy Engraulis mordax, has been fluctuating more smoothly without reaching extreme population densities, suggesting that direct environmental factors may not be solely responsible for the dynamics of the sardine. Similar patterns have been documented in Sardinops sagax over the whole Holocene period, with bouts of very low abundance spanning up to 500 years (De Vries and Pearcy, 1982); and in Sardinops melanistica on the timescale of the last four centuries (Tsuboi, 1984; Kondô, 1987). Tens and even hundreds of consecutive generations at either high or very low abundance may have given ample time for contrasting selective pressures to operate on traits related to competitive performance, and cause the alternations of high and low abundance observed in the data. The combination of multiple ecological equilibria and adaptive reversals between them offers an alternative explanation, previously suggested in a verbal model by Cury (1988), to purely ecological models of such longterm fluctuations between high and low density in fish communities (Ferrière and Cazelles, 1999). It would be interesting to re-analyse the subfossil fish scale data to investigate the occurrence of concomitant changes in fish body size, which could be indicative of density fluctuations driven by evolutionary reversals.

\subsection{Mechanism causing evolutionary reversals}

The biological mechanism causing evolutionary reversals involves two ingredients. First, there must exist trait values in the phenotypic space near which mutant phenotypes can invade and yet fail to go to fixation, while their transitory presence cause the resident population to switch between two ecological states. This phenomenon brings new evidence that the 'invasion-impliesfixation' principle of adaptive dynamics theory can be violated even for arbitrarily small mutations, and does require the uniqueness of the ecological attractor to hold true (Geritz et al., 1997). So far the few examples known to contradict the invasion-implies-fixation principle had been constructed by resorting to mutations of unlikely large effect (Doebeli (1998); Diekmann et al. (1999); De Feo 
and Ferrière (2000); Mylius and Diekmann (2001); see also Case (1995) and Abrams and Shen (1989) in the context of species invasion in communities).

The second key ingredient for evolutionary reversals is that selective pressures operate in opposite directions on the same phenotypes depending on whether the population density is high or low. Why this contrasting effect of selection arises in our model is easily understood: at high population density, a mutant is engaged in many competitive contests; thus, it has much to gain by investing more into competitive efficiency (through an increased trait value) at the expense of reducing its reproductive rate (provided that the trade-off between competition and reproduction is not too steep). In contrast, at low density encounters are few, there is little to be gained from improved competitive ability; thus, one can expect advantageous mutants to be characterized by a larger intrinsic reproductive rate, achieved with smaller phenotype.

Khibnik and Kondrashov (1997) also showed that cyclic evolution involving sharp transitions between multiple ecological attractors can occur in Red Queen dynamics of multispecies coevolution. Our study demonstrates that the coevolutionary Red Queen scenario is not necessary to explain the reversal of selective pressures between different ecological attractors and the endless variations in adaptive traits; purely intraspecific interactions are sufficient.

Matsuda and Abrams (1994a) also considered the evolution of body size under asymmetrical competition, but the uniqueness of ecological equilibrium at any trait value prevented the mechanism causing adaptive reversals to operate in their model. As a consequence, they could observe only the directional evolution of large body size and low population density, a process that could lead to population self-extinction. In contrast, if the conditions for adaptive reversals are met in our model, the evolution toward large phenotype terminates at a trait value where population density drops fast and dramatically whereas selective pressures revert and 'rescue' the population from extinction by promoting smaller phenotype and slowly increasing population density as a by-product. Taxon cycles driven by asymmetrical interspecific competition were proposed to explain the longterm maintenance of diversity in communities subject to colonization and coevolutionary-driven extinction (Rummel and Roughgarden, 1983, 1985; Taper and Case, 1992). Likewise, adaptive cycles driven by asymmetrical intraspecific competition can explain the long-term persistence of a single population subject to genetic variability (providing the mutants needed to beget switches between ecological equilibria) and selective reversals.

\subsection{Concluding remarks}

Any ecological scenario that makes the number of ecological attractors dependent upon an adaptive trait sets the stage for switches between population attractors driven by the evolutionary dynamics of the trait. This has been clearly recognized and exploited by Matsuda and Abrams (1994b) for showing that the evolution of anti-predator ability may result in self-extinction of a prey population. Here we have used the same principle to demonstrate that the occurrence of adaptive reversals and consequent population cycling is a likely property of ecological systems that possess alternative equilibria. We stress that this property is not bound to the assumption that multiple steady states are caused by a special ecological mechanism. A discounting function of the form of Holling type III response was chosen here to make the analysis mathematically tractable. Qualitatively similar results would be obtained with a Holling type II-like discounting function; the low-abundance stable equilibrium would simply be replaced with the zero equilibrium, thus making the 'evolutionary suicide' of the population almost unavoidable. Interestingly, some compelling evidence for evolutionary reversals of social traits has been gathered recently (Velicer et al., 1998; Hibbett et al., 2000). Sociality can induce an Allee effect that may be responsible for a discounting factor of the type considered here (Courchamp et al., 1999). Thus, adaptive switches between multiple ecological equilibria may point to a purely endogenous mechanism responsible for the repeated evolutionary rise and fall of social behavior. 
Our study delineates two ways in which selection may depend on density. In the first and classical case, each value of a trait uniquely determines the population density. If the density varies monotonically (e. g. decreases) with the trait value, the direction of adaptation may change with density as a mere consequence of directional selection toward an intermediate phenotype: at lower trait value, hence higher density, selection would favor an increase of the trait; at higher trait value, hence lower density, selection would promote the decrease of the trait. In contrast, when multiple equilibria are feasible, the direction and strength of selection may differ at the same trait value depending upon the state (e. g. high versus low density) on which the population is actually resting. Real systems possessing multiple equilibria should therefore be useful to set up laboratory experiments in which the effect of density on selection is strictly isolated.

\section{References}

Abrams, P. A., and Shen, L. 1989. Population dynamics of systems with consumers that maintain a constant ratio of intake rates of two resources. Theoretical Population Biology 35:51-89.

Baumgartner, T. R., Soutar, A., and Ferreira-Bartrina, V. 1992. Reconstruction of the history of Pacific sardine and northern anchovy populations over the past two millenia from sediments of the Santa Barbara basin, California. Californian Cooperative Oceanic Fishery Investment Report 33:24-40.

Blackburn, T. M., and Lawton, J. H. 1994. Population abundance and body size in animal assemblages. Philosophical Transactions of the Royal Society of London B 343:33-39.

Brauer, F. 1979. Harvesting strategies for population systems. Rocky Mountain Journal of Mathematics 9:19-26.

Brooks, J. L., and Dodson, S. I. 1965. Predation, body size, and composition of plankton. Science 150:28-35.

Brown, J. H., ed. 1995. Macroecology. The University of Chicago Press, Chicago.

Calder III, W. A. 1996. Size, Function, and Life History. Dover Publications Inc., Mineola, NY.

Case, T. J. 1995. Surprising behavior from a familiar model and implications for competition theory. The American Naturalist 146:961-966.

Champagnat, N., Ferrière, R., and Ben Arous, G. 2001. The canonical equation of adaptive dynamics: a mathematical view. Selection 2:73-83.

Connell, J. H., and Sousa, W. P. 1983. On the evidence needed to judge ecological stability or persistence. The American Naturalist 121:789-824.

Courchamp, F., Clutton-Brock, T., and Grenfell, B. 1999. Inverse density dependence and the allee effect. Trends in Ecology \& Evolution 14:405-410.

Crawley, M. J., ed. 1992. Natural Enemies. Blackwell Scientific Publications, Oxford, UK.

Cury, P. 1988. Pressions sélectives et nouveautés évolutives: une hypothèse pour comprendre certains aspects des fluctuations à long terme des poissons pélagiques côtiers. Canadian Journal of Fisheries and Aquatic Sciences 45:1099-1107.

De Feo, O., and Ferrière, R. 2000. Bifurcation analysis of invasion in a simple competition model: On-off intermittency and basin riddling. International Journal of Bifurcation and Chaos 10:443-452. 
De Vries, T. J., and Pearcy, W. G. 1982. Fish debris in sediments of the upwelling zone off central Peru: A late Quaternary record. Deep-Sea Research 28:87-109.

Dennis, B. 1989. Allee effects: Population growth, critical density, and the chance of extinction. Natural Resource Modelling 3:481-538.

Dieckmann, U., and Law, R. 1996. The dynamical theory of coevolution: A derivation from stochastic ecological processes. Journal of Mathematical Biology 34:579-612.

Diekmann, O., Mylius, S. D., and ten Donkelaar, J. R. 1999. Saumon à la kaitala et getz, sauce hollandaise. European Economic-Review 1:261-275.

Doebeli, M. 1998. Invasion of rare mutants does not imply their evolutionary success: A counterexample from metapopulation theory. Journal of Evolutionary Biology 11:389-401.

Doebeli, M., and Koella, J. 1995. Evolution of simple population dynamics. Proceedings of the Royal Society of London B 260:119-125.

Ebenman, M., Johanson, A., Jonsson, T., and Wennergren, U. 1996. Evolution of stable population dynamics through natural selection. Proceedings of the Royal Society of London B 263:1145-1151.

Ferrière, R., and Cazelles, C. 1999. Universal power laws govern intermittent rarity in communities of interacting species. Ecology 80:1505-1521.

Ferrière, R., and Fox, G. A. 1995. Chaos and evolution. Trends in Ecology \& Evolution 10:480485 .

Ferrière, R., and Gatto, M. 1993. Chaotic dynamics can result from natural selection. Proceedings of the Royal Society of London B 251:33-38.

- 1995. Lyapunov exponents and the mathematics of invasion in oscillatory or chaotic populations. Theoretical Population Biology 48:126-171.

Gaston, K. J., and Kunin, W. E. 1997. Rare-common differences: An overview. In W. E. Kunin and K. J. Gaston, eds., The Biology of Rarity, pages 12-29. Chapman \& Hall, London.

Gatto, M. 1993. The evolutionary optimallity of oscillatory and chaotic dynamics in simple population models. Theoretical Population Biology 43:310-336.

Geritz, S. A. H., Metz, J. A. J., Kisdi, E., and Meszéna, G. 1997. The dynamics of adaptation and evolutionary branching. Physical Review Letters 78:2024-2027.

Hanski, I. 1985. Single-species spatial dynamics may contribute to long-term rarity and commonness. Ecology 66:335-343.

Heino, M., Metz, J. A. J., and Kaitala, V. 1998. The enigma of frequency-dependent selection. Trends in Ecology \& Evolution 13:367-370.

Hibbett, D. S., Gilbert, L. B., and Donoghue, M. J. 2000. Evolutionary instability of ectomycorrhizal symbioses in basidiomycetes. Nature 407:506-508.

Holling, C. S. 1973. Resilience and stability of ecological systems. Annual Review of Ecology and Systematics 4:1-23.

Holt, R. D. 1997. Rarity and evolution: some theoretical considerations. In W. E. Kunin and K. J. Gaston, eds., The Biology of Rarity, pages 209-234. Chapman \& Hall, London. 
Huberman, G. 1978. Qualitative behavior of a fishery system. Mathematical Biosciences 42:1-14.

Khibnik, A. I., and Kondrashov, A. S. 1997. Three mechanisms of Red Queen dynamics. Proceedings of the Royal Society of London B 264:1049-1056.

Khibnik, A. I., Kuznetsov, Y. A., Levitin, V. V., and Nikolaev, E. V. 1993. Continuation techniques and interactive software forbifurcation analysis of ODEs and iterated maps. Physica D 62:360-370.

Kisdi, E. 1999. Evolutionary branching under asymmetric competition. Journal of Theoretical Biology 197:149-162.

Kondô, K. 1987. Relationships between long term fluctuations in the Japanese sardine (Sardinops melanosticus) and oceanographic conditions. International Symposium on Long term Changes in Marine Fish Populations, 18-21 November 1986, Vigo.

Kuznetsov, Y. A. 1998. Elements of Applied Bifurcation Theory. Springer Verlag, Berlin.

Lawton, J. H. 1989. What is the relationship between population density and body size in animals? Oikos 55:429-434.

Lenski, R. E., and Bennett, A. F. 1993. Evolutionary response of escherichia coli to thermal stress. The American Naturalist 142:S47-S64.

Marquet, P. A., Navarette, S. A., and Castilla, J. C. 1990. Scaling population density to body size in rocky intertidal communities. Science 250:1125-1127.

Matsuda, H., and Abrams, P. A. 1994a. Runaway evolution to self-extinction under asymmetrical competition. Evolution 48:1764-1772.

- 1994b. Timid consumers: Self extinction due to adaptive change in foraging and anti-predator effort. Theoretical Population Biology 45:76-91.

May, R. M. 1977. Thresholds and breakpoints in ecosystems with a multiplicity of stable states. Nature 269:471-477.

Metz, J. A. J., Nisbet, R. M., and Geritz, S. A. H. 1992. How should we define fitness for general ecological scenarios? Trends in Ecology \& Evolution 7:198-202.

Mueller, L. D. 1997. Theoretical and empirical examination of density-dependent selection. Annual Review of Ecology and Systematics 28:269-288.

Muratori, S., and Rinaldi, S. 1991. A separation condition for the existence of limit cycles in slow-fast systems. Applied Mathematical Modeling 61:312-318.

Mylius, S. D., and Diekmann, O. 2001. The resident strikes back: On the evolutionary jumping between population dynamical attractors. Journal of Theoretical Biology 211:297-311.

Navarette, S. A., and Menge, B. A. 1997. The body size-population density relationship in tropical rocky intertidal communities. Journal of Animal Ecology 66:557-566.

Noy-Meir, I. 1975. Stability of grazing systems: An application of predator-prey graphs. Journal of Ecology 63:459-483.

Orians, G. H. 1997. Evolved consequences of rarity. In W. E. Kunin and K. J. Gaston, eds., The Biology of Rarity, pages 190-208. Chapman \& Hall, London. 
Persson, L. 1985. Asymmetrical competition: are larger animals competitively superior? The American Naturalist 126:261-266.

Persson, L., Leonardsson, K., de Roos, A. M., Gyllenberg, M., and Christensen, B. 1998. Ontogenetic scaling of foraging rates and the dynamics of a size-structured consumer-resource model. Theoretical Population Biology 54:270-293.

Pimentel, D. 1968. Population regulation and genetic feedback. Science 159:1432-1437.

Rand, D. A., Wilson, H. B., and McGlade, J. M. 1994. Dynamics and evolution: Evolutionarily stable attractors, invasion exponents and phenotype dynamics. Philosophical Transactions of the Royal Society of London B 1305:261-283.

Rinaldi, S., and Scheffer, M. 2000. Geometric analysis of ecological models with slow and fast processes. Ecosystems 3:507-521.

Rosenzweig, M. L. 1995. Species Diversity in Space and Time. Cambridge University Press, Cambridge, UK.

Rummel, J. D., and Roughgarden, J. 1983. Some differences between invasion-structured and coevolution-structured competitive communities: A preliminary theoretical analysis. Oikos 41:477-486.

—. 1985. The theory of faunal build-up for competition communities. Evolution 39:1009-1033.

Scheffer, M., Carpenter, S., Foley, J. A., Folke, C., and Walker, B. 2001. Catastrophic shifts in ecosystems. Nature 413:591-596.

Schwinning, S., and Fox, G. A. 1995. Population dynamic consequences of competitive symmetry in annual plants. Oikos 72:422-432.

Soutar, A., and Isaacs, J. D. 1974. Abundance of pelagic fish during the $19^{\text {th }}$ and $20^{\text {th }}$ centuries as recorded in anaerobic sediment off California. Fishery Bulletin 72:257-274.

Southwood, T. R. E., and Comins, H. N. 1976. A synoptic population model. Journal of Animal Ecology 45:949-965.

Stephens, P. A., and Sutherland, W. J. 1999. Consequences of the Allee effect for behaviour, ecology and conservation. Trends in Ecology \& Evolution 14:401-405.

Taper, M. L., and Case, T. J. 1992. Coevolution among competitors. In D. Futuyma and J. Antonovics, eds., Oxford Surveys in Evolutionary Biology, volume 8, pages 63-111. Oxford University Press, Oxford.

Travis, J. 1990. The interplay of population dynamics and the evolutionary process. Philosophical Transactions of the Royal Society of London B 330:253-259.

Travis, J., Keen, W. H., and Juilianna, J. 1985. The effects of multiple factors on viability selection in Hyla gratiosa tadpoles. Evolution 39:1087-1099.

Tsuboi, M. 1984. Marine resources trends of purse seines — as main aims of sardine and mackerel fisheries - economical structure of purse seine fishery. Progress Report of Synthesis Investigations of the Japanese Fisheries, 1983 Project. Dai-Nihon Suisankai, March 1984. Fishery Bulletin . 
Velicer, G., Kroos, L., and Lenski, R. E. 1998. Loss of social behaviors by myxococcus xanthus during evolution in an unstructured habitat. Proceedings of the National Academy of Science USA 95:12376-12380.

Wilbur, H. M. 1984. Complex life cycles and community organization in amphibians. In P. W. Price, C. N. Slobodchikoff, and W. S. Gaud, eds., A New Ecology: Novel Approaches to Interactive Systems, pages 196-224. John Wiley \& Sons, New York.

Wilson, D. S. 1975. The adequacy of body size as a niche difference. The American Naturalist 109:769-784. 


\section{Appendix}

\subsection{Condition for ecological bistability}

The ecological model is given by eq. (1) with $r(u)=r_{0} \exp (-\gamma u)$, where $r_{0}$ and $\gamma$ are constant parameters. In the $(u, a)$ parameter plane, the transition from one stable positive equilibrium to three positive equilibria (two stable, one unstable) corresponds to a so-called fold bifurcation, and two fold bifurcation curves determine two values of $u\left(u_{l}\right.$ and $\left.u_{h}\right)$ for each value of $a$ within an appropriate range. These two curves meet at a single point (called cusp) which identifies the $a$ value beyond which no bistability can occur. By writing the standard conditions for fold bifurcations (e. g., Kuznetsov, 1998)), we find

$$
u_{l}=-\frac{1}{\gamma} \ln \left[\frac{x_{l}}{r_{0}}\left(\frac{c}{x_{l}^{2}+d^{2}}+a\right)\right] \quad u_{h}=-\frac{1}{\gamma} \ln \left[\frac{x_{h}}{r_{0}}\left(\frac{c}{x_{h}^{2}+d^{2}}+a\right)\right]
$$

where

$$
x_{l}=\left[\frac{c-2 a d^{2}-\sqrt{c\left(c-8 a d^{2}\right)}}{2 a}\right]^{1 / 2} \quad x_{h}=\left[\frac{c-2 a d^{2}+\sqrt{c\left(c-8 a d^{2}\right)}}{2 a}\right]^{1 / 2}
$$

The cusp point is given by

$$
u=-\frac{1}{\gamma} \ln \left[\frac{8 d r_{0}}{3 \sqrt{3} c}\right] \quad a=\frac{c}{8 d^{2}}
$$

\subsection{Condition for adaptively-driven population cycles}

The selection gradient is $s(u, x)=-r_{0} \gamma \exp (-\gamma u)+\beta x$. Hence the selection null-isocline in the $(u, x)$ space is:

$$
x=\frac{r_{0} \gamma}{\beta} \exp (-\gamma u)
$$

Adaptively-driven population cycles occur if and only if the selection null-isocline separates the upper branch of high population equilibria, and the lower branch of low population equilibria. Elementary algebra yields the following necessary conditions:

$$
\begin{array}{cc}
\frac{r_{0} \gamma}{\beta} \exp \left(-\gamma u_{l}\right)>x_{l} & \frac{r_{0} \gamma}{\beta} \exp \left(-\gamma u_{h}\right)<x_{h} \\
\frac{r_{0} \gamma}{\beta} \exp \left(-\gamma u_{l}\right)<\frac{d^{2} r\left(u_{l}\right)}{a x_{l}^{2}} & \frac{r_{0} \gamma}{\beta} \exp \left(-\gamma u_{h}\right)>\frac{d^{2} r\left(u_{h}\right)}{a x_{h}^{2}}
\end{array}
$$

and systematic numerical computations confirm that these conditions are also sufficient. The surface and curves displayed in Figure 4 are computed from these conditions.

\subsection{Numerical analysis}

We used the following asymmetric competition function:

$$
b\left(u, u^{\prime}\right)=2 a\left\{1-\left[1+\exp \left(\delta\left(u^{\prime}-u\right)\right)\right]^{-1}\right\}
$$

The strength of competitive asymmetry is measured by $\beta=a \delta / 2$. Notice that for all $u, b(u, u)=$ $a$. Numerical bifurcation analysis were carried out by making use of the software LOCBIF (Khibnik et al., 1993). 